\title{
High apolipoprotein M serum levels correlate with chronic obstructive pulmonary disease
}

\author{
Hui Li ${ }^{1}$, Yinyin Liư', Ling Wang ${ }^{1}$, Ting Shen ${ }^{1}$, Wenhan Du', Zhijun Liu², Ruohong Chen ${ }^{1}$ and Min $\mathrm{Hu}^{1 *}$
}

\begin{abstract}
Background: Recently, variations in a component of high-density lipoprotein (HDL), namely apolipoprotein M (apoM), were found to be associated with chronic obstructive pulmonary disease (COPD). The aim of this study was to evaluate the association between apoM and COPD severity. Factors associated with apoM, COPD, or coronary artery disease (CAD) were also assessed.
\end{abstract}

Methods: A total of 110 COPD patients and 110 age- and sex-matched non-COPD controls were included. Among them, thirty COPD patients and seven non-COPD controls had CAD. ApoM and pentraxin-3 levels were measured by ELISA. Additionally, the levels of high-sensitivity C-reactive protein (hs-CRP), cholesterol, and triglyceride were assessed using an automatic biochemical analyzer.

Results: Serum apoM levels increased gradually with COPD severity, with the most prominent apoM elevation observed in very severe COPD cases. In addition, ApoM was correlated with percent-predicted forced expiratory volume in one second (\% predicted FEV1) $(r=-0.38$,

$P<0.001)$, low-density lipoprotein cholesterol (LDL-C) $(r=0.23, P<0.017)$, and hs-CRP $(r=0.24, P=0.01)$ in COPD patients. Furthermore, apoM was shown to be a risk factor for COPD onset $(\mathrm{OR}=1.095,95 \% \mathrm{Cl}=1.034-1.160$, $P=0.002)$, but not associated with CAD in COPD patients.

Conclusions: Serum apoM was elevated in COPD patients and increased gradually with COPD severity. However, there was no association between apoM and CAD development in COPD patients.

Keywords: Apolipoprotein M, Chronic obstructive pulmonary disease, Forced expiratory volume in one second, $\%$ predicted $\mathrm{FEV}_{1}$, Cardiovascular disease

\section{Background}

Chronic obstructive pulmonary disease (COPD), the fourth leading cause of morbidity and mortality worldwide, is associated with significant economic and social burden [1]. An increasing number of COPD patients die from systemic inflammatory complications, such as cardiovascular diseases, rather than respiratory system failure [2, 3]. However, the mechanisms linking systemic inflammation to cardiovascular diseases remain unclear and cannot simply be explained by the effects of smoking or hypertension [3].

\footnotetext{
*Correspondence: minhu013@sina.com

'Department of Clinical Laboratory, The Second Xiangya Hospital, Centre

South University, Changsha 410011, Hunan Province, China

Full list of author information is available at the end of the article
}

Apolipoprotein M (apoM) is a $26-\mathrm{kDa}$ apolipoprotein that belongs to the lipocalin protein superfamily. In the serum, apoM mainly binds to high-density lipoproteins (HDL). ApoM-HDL interactions promote pre- $\beta$ HDL formation [4] and increase cholesterol efflux from foam cells [5]. Moreover, the anti-atherosclerotic properties of apoM are attributed to its anti-inflammatory activity [6] and protect low-density lipoprotein cholesterol (LDL-C) against oxidation [7]. ApoM can potentially protect the endothelium under homeostatic conditions [8] and suppress lymphopoiesis and neuroinflammation during the adaptive immune response $[9,10]$.

The human apoM gene is located in the major histocompatibility complex class III region of chromosome 6, which 
also contains several genes involved in immune and inflammatory responses [11]. Recent studies suggested that a number of inflammatory factors might be correlated with increased serum apoM levels in COPD patients $[12,13]$. Interestingly, serum and lung platelet-activating factor and leptin levels are increased in COPD patients and correlated with disease severity [14, 15], suggesting that the systemic inflammation associated with COPD might cause an increase in apoM expression. As part of the MESA COPD study, Burkart et al. [16] recently reported that two single nucleotide polymorphisms flanking the apoM gene are associated with altered lung function. The latter study also revealed that apoM mRNA is inversely associated with the $\mathrm{FEV}_{1} / \mathrm{FVC}$ ratio [16].

The associations between apoM expression and markers of inflammation remain unknown in COPD patients. We hypothesized that serum apoM levels correlate with the onset and severity of COPD. The main purpose of this study was to assess the serum apoM level and determine its clinical associations with lung function and inflammatory markers in a cohort of patients with COPD at different stages of disease severity.

\section{Methods}

\section{Subjects}

This was a case-control study of 110 COPD patients evaluated at The Second Xiangya Hospital between July 2014 and June 2015. The diagnosis of COPD was based on each patient's clinical history, physical examination, chest radiography, arterial blood gas measurements, and lung function, according to the Global Initiative for Chronic Obstructive Lung Disease (GOLD) 2015 classification [17]. Patients in this study were classified as GOLD stages I/II $(n=32)$, III $(n=39)$, and IV $(n=39)$.

The inclusion criterion was COPD patients with an $\mathrm{FEV}_{1} / \mathrm{FVC}<0.7$. Exclusion criteria were: 1 ) body mass index (BMI) $>30 \mathrm{~kg} / \mathrm{m}^{2}$; 2) history of pulmonary embolism; 3) metabolic acidosis; 4) immunological disease; 5) cancer; 6) venous or arterial thrombosis; 7) renal disease.

Coronary artery disease (CAD) was diagnosed by the presence of stenosis on coronary angiography. Hypertension was defined as systolic blood pressure $\geq 140 \mathrm{mmHg}$ and/or diastolic blood pressure $\geq 90 \mathrm{mmHg}$. Diabetes mellitus was defined as fasting plasma glucose $\geq 7.0 \mathrm{mmol} / \mathrm{L}$ and 2-h glucose tolerance test glucose $>11.1 \mathrm{mmol} / \mathrm{L}$.

The control group included 110 age and sex matched non-COPD volunteers who underwent physical examination in our hospital, with $\mathrm{FEV}_{1} / \mathrm{FVC}<0.7$ and a normal chest X-ray.

The study protocol was reviewed and approved by The Second Xiangya Hospital Investigational Review Board. Written informed consent was obtained from all participants.

\section{Blood sampling}

After an overnight fast and at least $20 \mathrm{~min}$ of rest, blood samples were collected from each subject. Serum was obtained by centrifugation at $3500 \mathrm{rpm}$ for $5 \mathrm{~min}$, and aliquots were stored at $-80^{\circ} \mathrm{C}$.

\section{Enzyme-linked immunosorbent assay (ELISA) for apoM assessment}

Serum apoM levels were measured using a sandwich ELISA (Yuan Tai Bio Inc., Changsha, Hunan, People's Republic of China). Optical density (OD) was measured at $450 \mathrm{~nm}$ (with a background reading at $620 \mathrm{~nm}$ ) on an ELX-800 absorbance reader (BioTek Instruments, Inc., Winooski, VT, USA). The concentration of apoM (as $\mathrm{mg} / \mathrm{L}$ ) in each sample was derived from a standard curve.

\section{Lipoprotein and inflammation marker levels}

Serum triglyceride, total cholesterol, HDL cholesterol (HDL-C), LDL-C, apolipoprotein A (apoA), apolipoprotein $\mathrm{B}$ (apoB), lipoprotein(a), and high-sensitivity C-reactive protein (hs-CRP) levels were measured on an ARCHITECT c8000 System (Abbott Laboratories, Irving, TX, USA). Pentraxin-3 levels were detected using an ELISA kit (CUSABIO, Wuhan, China) according to the manufacturer's instructions.

\section{Statistical analysis}

Continuous data are presented as the mean \pm standard deviation; categorical data are expressed as percentages. Continuous data were analyzed by Student's $t$-test or one-way analysis of variance (ANOVA) with Tukey's test, as appropriate. Variables with a skewed distribution were converted by logarithmic transformation before analysis. Pearson's correlation analysis was used to assess the associations between the apoM level and normally distributed variables. Spearman correlation analysis was used for variables with skewed distributions. Binary logistic regression models were carried out to identify the risk factors for COPD onset and identify the risk factors for CAD in COPD patients. All analyses were performed with SPSS 20.0 (SPSS, USA) or GraphPad Prism 5.0 (GraphPad Software, La Jolla, CA, USA). Two-sided $P<0.05$ was considered statistically significant.

\section{Results}

\section{Patient characteristics}

Figure 1 depicts the patient flowchart. From 187 COPD patients screened for participation, 32 did not meet the eligibility criteria and 45 refused to participate; therefore, 110 patients were finally assessed. Additionally, 254 controls were screened for participation; 45 failed the eligibility criteria, 61 refused to participate, and 28 were eliminated by the matching criteria; therefore, 110 were finally included. 


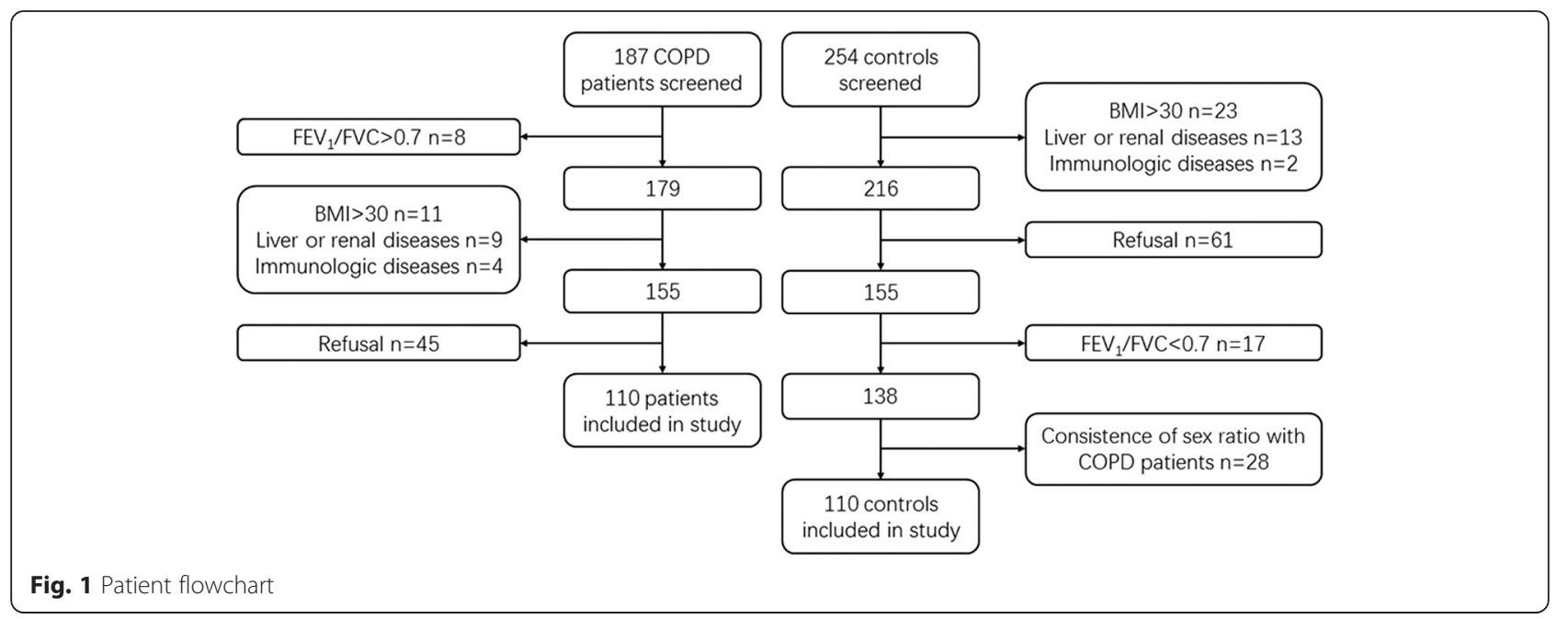

Age $(67 \pm 10$ vs. $67 \pm 10$ years, $P=0.823)$, gender ( $86 \%$ vs. $86 \%$ men, $P=0.99)$, and BMI $(22.2 \pm 2.3$ vs. $22.2 \pm 2.3 \mathrm{~kg} / \mathrm{m}^{2}, P=0.954$ ) were similar between the COPD and control groups (Table 1) and across all COPD GOLD stages (Additional file 1: Table S1). The COPD group had a higher proportion of smokers $(64 \%$ vs. $35 \%, P<0.001)$, CAD ( $28 \%$ vs. $6 \%, P<0.001)$, hypertension ( $25 \%$ vs. $11 \%, P=0.008)$, and hormone therapy (27\% vs. $0 \%, P<0.001)$ compared with the control group. \% predicted $\mathrm{FEV}_{1}$ and $\mathrm{FEV}_{1} / \mathrm{FVC}$ were lower in COPD patients compared with control values $(P<0.001)$. Pentraxin-3 levels were higher in COPD patients compared with the control group $(1.28 \pm 2.66$ vs. $0.96 \pm 2.05 \mathrm{ng} / \mathrm{L}, P=0.002)$.

\section{Serum apoM amounts are elevated in COPD patients}

Serum apoM levels in COPD patients were $23.08 \pm$ $9.54 \mathrm{mg} / \mathrm{L}$, higher than values obtained for healthy controls $(P<0.0001$; Table 1$)$. The most pronounced apoM increase in COPD patient subpopulations was observed in GOLD stage IV, followed by stages III and I/II $(P<0.05$; Fig. 2).

\section{Factors associated with apoM in COPD patients}

The serum lipid profile and pulmonary function were assessed for their associations with apoM using univariate correlation analyses; \% predicted $\mathrm{FEV}_{1}$ was used to evaluate lung function. Interestingly, \% predicted $\mathrm{FEV}_{1}$ was inversely correlated with the serum apoM level $(r=-0.38, P<0.001$; Fig. 3a). ApoM was positively correlated with serum LDL-C $(r=0.23, P=0.017$; Fig. $3 \mathrm{~b})$ and hs-CRP $(r=0.24, P=0.01$; Fig. 3c). Marginally significant correlations were observed between apoM and pentraxin-3 $(r=0.18, P=0.06$; Fig. $3 \mathrm{~d})$.

\section{Risk factors for COPD onset}

To identify the risk factors associated with the onset of COPD symptoms, a binary logistic regression model of the whole study population was built by initially including all variables with $P<0.05$ in univariate analyses (Table 1 ) and systematically excluding variables that were not significant $(P>0.10)$. Triglycerides $(\mathrm{OR}=0.528,95 \% \mathrm{CI}=0.283$ $0.985, P=0.045)$ and HDL-C $(\mathrm{OR}=0.292,95 \% \mathrm{CI}=0.101$ $0.844, P=0.023)$ were identified as protective factors. In contrast, apoM $(\mathrm{OR}=1.095,95 \% \mathrm{CI}=1.034-1.160$, $P=0.002)$, smoking history $(\mathrm{OR}=2.542,95 \% \mathrm{CI}=1.188$ 5.440, $P=0.016)$, and hs-CRP $(\mathrm{OR}=1.249,95 \% \mathrm{CI}=$ 1.136-1.374, $P<0.001$ ) were found to be risk factors for COPD onset in the final model (Table 2).

\section{Risk factors for CAD in COPD patients}

To identify the risk factors associated with CAD in COPD patients, a binary logistic regression model was performed. Non-significant variables $(P>0.05)$ were systematically excluded. Use of a $\beta-2$ adrenergic receptor antagonist $(\mathrm{OR}=0.179,95 \% \mathrm{CI}=0.040-0.804, P=0.025)$ was identified as a protective factor. In contrast, hypertension $(\mathrm{OR}=2.018,95 \% \mathrm{CI}=1.380-2.952, P<0.001)$ and aminophylline use $(\mathrm{OR}=7.048,95 \% \mathrm{CI}=2.169-22.906$, $P=0.001$ ) were risk factors for CAD onset in COPD patients (Table 3). In this model, apoM was not associated with the development of CAD in COPD patients.

\section{Discussion}

This study revealed a relationship between the serum apoM level and \% predicted $\mathrm{FEV}_{1}$, a gold standard for severity assessment of airflow obstruction. These results extend the findings of the MESA COPD study, which demonstrated an inverse relationship between airflow obstruction and apoM mRNA levels and suggested that serum apoM is a predictor of COPD onset [16]. As shown above, serum apoM was not associated with CAD in COPD patients. A potential mechanism behind the high cardiovascular disease prevalence in COPD patients is the altered endothelium function resulting from airflow obstruction [18]. 
Table 1 Demographic and biochemical parameters of the study subjects

\begin{tabular}{|c|c|c|c|}
\hline & COPD & Controls & $P$ value \\
\hline Subjects, n & 110 & 110 & \\
\hline \multicolumn{4}{|l|}{ Demographics } \\
\hline Age, years & $67 \pm 10$ & $67 \pm 10$ & 0.823 \\
\hline Sex, $\%$ male & 86 & 86 & 0.847 \\
\hline Body mass index, $\mathrm{kg} / \mathrm{m}^{2}$ & $22.2 \pm 2.3$ & $22.2 \pm 2.3$ & 0.954 \\
\hline Smoking history, \% & 64 & 35 & $<0.001$ \\
\hline \multicolumn{4}{|l|}{ GOLD stages, n } \\
\hline GOLD I/II & 32 & & \\
\hline GOLD III & 39 & & \\
\hline GOLD IV & 39 & & \\
\hline \multicolumn{4}{|l|}{ Comorbidities, \% } \\
\hline CAD & 28 & 6 & $<0.001$ \\
\hline Hypertension & 25 & 11 & 0.008 \\
\hline Diabetes mellitus & 5 & 1 & 0.098 \\
\hline \multicolumn{4}{|l|}{ Treatment, \% } \\
\hline Prednisone & 27 & 0 & $<0.001$ \\
\hline Aminophylline & 23 & 0 & $<0.001$ \\
\hline $\begin{array}{l}\beta-2 \text { adrenergic receptor } \\
\text { antagonist }\end{array}$ & 22 & 7 & 0.017 \\
\hline Anticholinergics & 23 & 0 & $<0.001$ \\
\hline \multicolumn{4}{|l|}{ Lung function } \\
\hline $\mathrm{FEV}_{1} \%$ predicted & $43.81 \pm 19.77$ & $92.34 \pm 8.29$ & $<0.001$ \\
\hline $\mathrm{FEV}_{1} / \mathrm{FVC}$ & $49.44 \pm 15.96$ & $97.48 \pm 3.73$ & $<0.001$ \\
\hline \multicolumn{4}{|l|}{ Lipids profile } \\
\hline Triglycerides, mmol/L & $1.17 \pm 0.67$ & $1.43 \pm 1.11$ & 0.036 \\
\hline Total cholesterol, $\mathrm{mmol} / \mathrm{L}$ & $4.16 \pm 0.82$ & $4.49 \pm 0.76$ & 0.003 \\
\hline $\mathrm{HDL}-\mathrm{C}, \mathrm{mmol} / \mathrm{L}$ & $1.15 \pm 0.30$ & $1.31 \pm 0.26$ & $<0.001$ \\
\hline $\mathrm{LDL}-\mathrm{C}, \mathrm{mmol} / \mathrm{L}$ & $2.50 \pm 0.77$ & $2.61 \pm 0.70$ & 0.285 \\
\hline Apolipoprotein A, g/L & $1.36 \pm 0.29$ & $1.46 \pm 0.27$ & 0.011 \\
\hline Apolipoprotein B, g/L & $0.89 \pm 0.22$ & $0.94 \pm 0.27$ & 0.122 \\
\hline Apolipoprotein M, mg/L & $23.08 \pm 9.54$ & $17.02 \pm 4.74$ & $<0.001$ \\
\hline Lipoprotein(a), g/L & $163.9 \pm 2.4$ & $99.0 \pm 9.3$ & $<0.001$ \\
\hline \multicolumn{4}{|l|}{ Inflammatory factors } \\
\hline $\mathrm{Hs}-\mathrm{CRP}, \mathrm{mg} / \mathrm{L}$ & $11.52 \pm 3.97$ & $1.47 \pm 2.77$ & $<0.001$ \\
\hline Pentraxin-3, ng/L & $1.28 \pm 2.66$ & $0.96 \pm 2.05$ & 0.002 \\
\hline
\end{tabular}

Data are mean \pm standard deviation. Values of triglycerides, lipoprotein(a), and hs-CRP were converted into a logarithmic form before analysis. The $p$-values refer to comparisons between the COPD and control groups GOLD Global Initiative for Chronic Obstructive Lung Disease, COPD chronic obstructive pulmonary disease, $C A D$ coronary artery disease, $H D L-C$ high-density lipoprotein cholesterol, LDL-C low-density lipoprotein cholesterol, $h s-C R P$ high-sensitivity C-reactive protein, $F E V$, forced expiratory volume in one second, FVC forced vital capacity, NS non-significant

Previous studies demonstrated that the type or degree of endothelial damage in COPD patients might be involved in the development of CAD $[19,20]$, but the exact

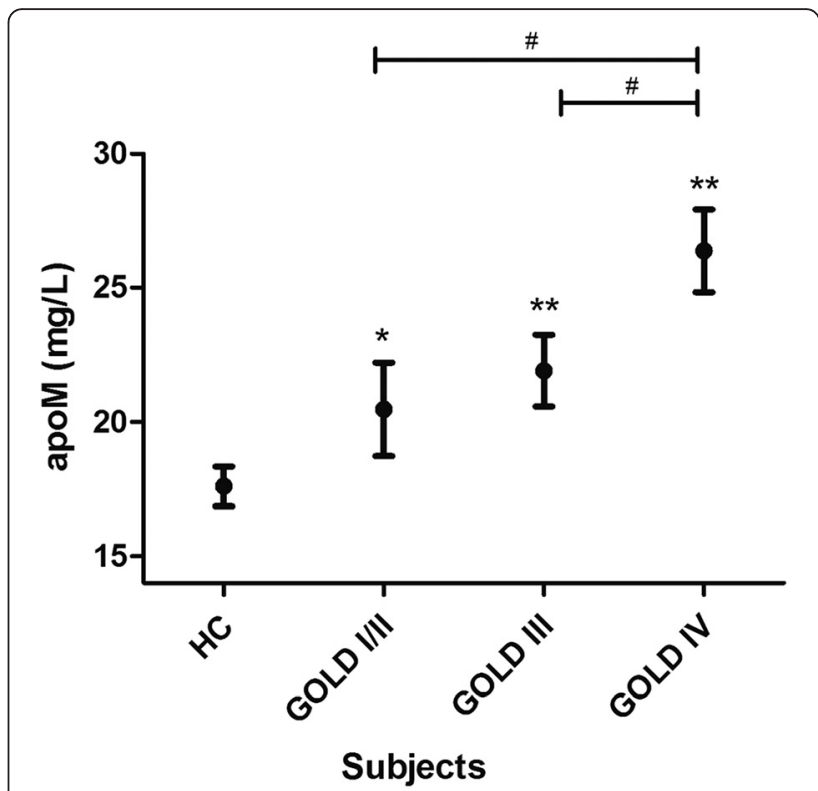

Fig. 2 Serum apolipoprotein M (apoM) is elevated in chronic obstructive pulmonary disease (COPD) patients. Serum concentrations of apoM in COPD patients, grouped according to the Global Initiative for Chronic Obstructive Lung Disease (GOLD) stage, and healthy control subjects (HC). Error bars represent the standard errors of the mean. ${ }^{*} P<0.05$, ${ }^{* *} P<0.01$ vs. HC. ${ }^{\#} P<0.05$, between two groups indicated by the horizontal line

interrelationships of CAD, COPD, and apoM remain elusive and require further investigation. Nevertheless, a previous study showed that apoM gene expression is inversely correlated with the severity of COPD [16].

Previously, apoM was considered a negative acute response protein [21]. ApoM gene expression is decreased in systemic inflammation stimulated by lipopolysaccharides (LPS), zymosan, or turpentine, as well as in patients with sepsis $[21,22]$. In this study, the apoM level was correlated with the amount of hs-CRP, which is a marker of systemic inflammation, but not with that of pentraxin-3, a marker of acute-phase immune reactions [23]. Many reasons may explain this discrepancy. The previous studies showing decreased apoM gene expression after exposure to chemicals or LPS were performed in an acute setting, using compounds known to elicit a massive systemic inflammatory response $[21,22]$. In this study, the increased inflammatory state was the result of a chronic state that might have different mechanisms compared to the acute state [24]. In addition, two different protective mechanisms involving apoM take place under acute or chronic inflammatory conditions. In response to acute inflammation, circulating apoM is severely depleted upon binding to S1P and the endothelium-protective S1P1 receptor; these interactions prevent an increase in vascular permeability or leakiness $[8,25]$. On the other hand, in the process of chronic systemic inflammation, apoM expression might be 

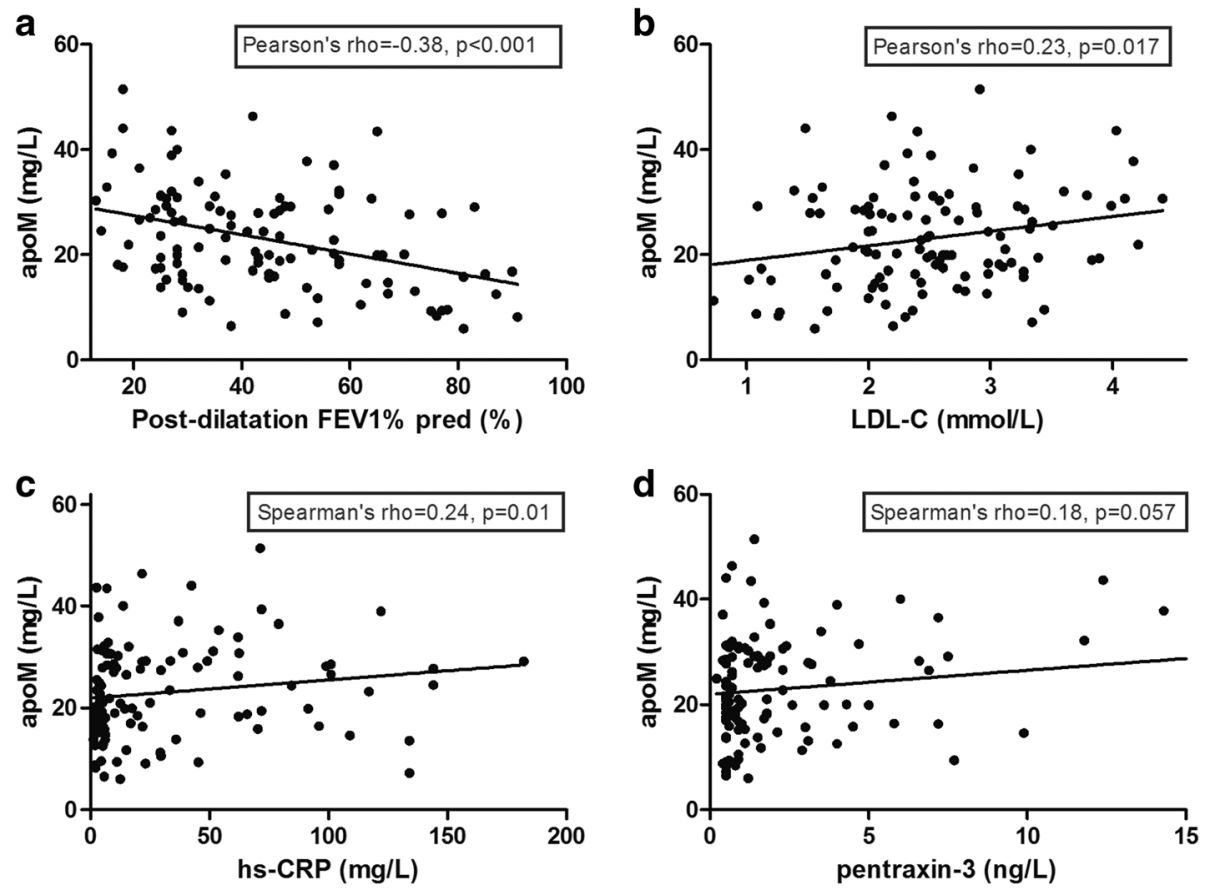

Fig. 3 Associations of apoM and \% predicted FEV $\mathrm{F}_{1}, \mathrm{LDL}-\mathrm{C}$, hs-CRP, and Pentraxin-3. a Post-dilatation forced expiratory volume in one second, \% predicted FEV 1 , was correlated with apoM ( $r=-0.38, P<0.001)$; and (b) low-density lipoprotein cholesterol (LDL-C) was correlated with apoM $(r=0.23, P=0.017)$. $\mathbf{c}$ High-sensitivity C-reactive protein (hs-CRP) was associated with apoM $(r=0.24, P=0.01)$. d Pentraxin-3 was not associated with apoM $(r=0.18, P=0.057)$

compensated or even up-regulated to counteract inflammatory effects over a longer period of time as part of an innate protective mechanism [26]. A previous study showed that elevated apoM gene expression is associated with lower $\mathrm{FEV}_{1} / \mathrm{FVC}$ [16], corroborating our findings. Nevertheless, the present study was not designed to determine the the mechanisms that cause apoM elevation in COPD patients, and additional investigations are needed for this purpose. A previous study revealed elevated pentraxin-3 in patients with obstructive sleep apnea and increased carotid intima-media thickness [23], in agreement with the present study; however, additional studies

Table 2 Binary logistic regression analysis for COPD

\begin{tabular}{|c|c|c|c|}
\hline & $P$ value & OR & $95 \% \mathrm{Cl}(\mathrm{OR})$ \\
\hline Hs-CRP & $<0.001$ & 1.249 & $1.136-1.374$ \\
\hline Smoking history & 0.016 & 2.542 & $1.188-5.440$ \\
\hline Triglycerides & 0.045 & 0.528 & $0.283-0.985$ \\
\hline HDL-C & 0.023 & 0.292 & $0.101-0.844$ \\
\hline Apolipoprotein A & 0.063 & 5.830 & $0.911-37.293$ \\
\hline Apolipoprotein M & 0.002 & 1.095 & $1.034-1.160$ \\
\hline
\end{tabular}

are warranted to characterize the inflammatory response in COPD patients and its relationship with atherosclerosis.

In this study, surprisingly, serum apoM was only associated with one lipid, LDL-C. Serum apoM reportedly shows positive correlations with serum total cholesterol, LDL-C, and HDL-C $[7,11]$. This is particularly true for HDL-C, as approximately $96 \%$ of apoM is bound to HDL particles [27]. In COPD patients, it is possible that apoM is readily redistributed within the pool of serum lipoproteins, and this occurs even though it is anchored to the surface of lipoproteins by its hydrophobic signal peptide [28]. ApoM was shown to be exchanged from HDL-C to LDL-C because of altered HDL-C amounts under inflammatory conditions $[29,30]$. Accordingly, in this study, HDL-C was lower in COPD patients. On the other hand, LDL-C particles are susceptible to oxidative modifications [31] and a decrease in particle size [32] during infection and inflammation, all of which increase the likelihood of their participation in the

Table 3 Binary logistic regression analysis for CAD in COPD patients

\begin{tabular}{llll}
\hline & $P$ & OR & $95 \%$ Cl (OR) \\
\hline Hypertension & $<0.001$ & 2.018 & $1.380-2.952$ \\
Aminophylline use & 0.001 & 7.048 & $2.169-22.906$ \\
$\beta-2$ adrenergic receptor antagonist use & 0.025 & 0.179 & $0.040-0.804$ \\
\hline
\end{tabular}

COPD chronic obstructive pulmonary disease, $C A D$ coronary artery disease 
development of atherosclerosis [33]. Indeed, small-sized LDL-C particles (known as subclass pattern B) more effectively cross the endothelial barrier [34] and are more susceptible to oxidative modifications [35]. This could result in a rapid uptake and accumulation of cholesterol in macrophages and, consequently, contribute to the development of atherosclerosis [36], which should be examined in future studies.

Finally, the abnormal lipid profile observed in this study suggests a high risk for cardiovascular diseases in COPD patients. Indeed, HDL-C is inversely associated with cardiovascular disease diagnosis [37, 38]; this is attributed primarily to the role of HDL-C in the reverse transport of cholesterol [39]. Increased $\mathrm{Lp}(\mathrm{a})$ is another strong independent risk factor for CAD [40]. The interaction of $\mathrm{Lp}(\mathrm{a})$ with pro-inflammatory oxidized phospholipids is thought to be atherogenic [41]. The decreased HDL-C levels and increased Lp(a) levels observed in the current study suggest an increased risk of cardiovascular in COPD patients. Systemic inflammation is strongly associated with atherosclerosis [36] and might explain the high risk of cardiovascular diseases associated with the pathological process of COPD.

A few limitations of the present study should be mentioned. The main limitation is the disproportionate number of men included in the study population. Women only accounted for $14 \%$ of all subjects. A study design with an approximately equal number of male and female subjects would provide more conclusive results. Another limitation is the relatively small number of COPD patients with CAD. Additional studies with a larger sample size for this COPD subpopulation will be necessary to elucidate the possible role of apoM in cardiac function. In this study, apoM was not associated with CAD when considering the use of drugs in the multivariate model. Longitudinal studies could be particularly helpful in determining the causal relationships of apoM, COPD, and CAD.

\section{Conclusions}

Serum apoM was elevated in COPD patients, and it increased gradually with the stage of COPD severity. It was also found to be an independent risk factor for COPD development. There was no association between apoM and the development of CAD in COPD patients.

\section{Ethics approval and consent to participate}

This study protocol was reviewed and approved by The Second Xiangya Hospital Investigational Review Board. Written informed consent was obtained from all participants.

\section{Consent for publication \\ Not applicable.}

\section{Availability of data and materials}

The dataset supporting the conclusions of this article is included within the article and its additional file.

\section{Additional file}

Additional file 1: Table S1. Clinical characteristics of COPD patients by GOLD stage. (DOC 38 kb)

\section{Abbreviations}

ANOVA: analysis of variance; apoB: apolipoprotein B; apoM: apolipoprotein M; CAD: coronary artery disease; COPD: chronic obstructive pulmonary disease: CRP: C-reactive protein; $\mathrm{FEV}_{1}$ : forced expiratory volume in one second; FVC: forced vital capacity; GOLD: Global Initiative for Chronic Obstructive Lung Disease; HDL: high-density lipoprotein; HDL-C: high-density lipoprotein cholesterol; hs-CRP: high-sensitivity C-reactive protein; LDL-C: low-density lipoprotein cholesterol; NS: non-significant;

OD: optical density.

\section{Competing interests}

The authors declare that they have no competing interests.

\section{Authors' contributions}

$H L$ carried out the study design, performed data collection and analysis, and wrote the manuscript. YL searched patients' information. LW and TS carried out the enzyme-linked immunosorbent assays. WD participated in the statistical analysis. ZL assessed lung function in patients. RC measured lipid concentrations. $\mathrm{MH}$ provided the funding support, conceived of the study, participated in its design and coordination, and provided critical revision. All authors read and approved the final manuscript.

\section{Acknowledgments}

We thank Mr. Li Weiping and Xu Fei for assistance with the statistical analysis.

\section{Funding}

The study was supported by the Independent Innovation Project Funds of the Central South University (2015zts314) and the Science and Technology Innovation Investment Programs of the Development and Reform Commission of Hunan Province ([2014] No 658 document, the $25^{\text {th }}$ plan of Central South University).

\section{Author details}

${ }^{1}$ Department of Clinical Laboratory, The Second Xiangya Hospital, Centre South University, Changsha 410011, Hunan Province, China. ${ }^{2}$ Department of Pulmonary Medicine, The Second Xiangya Hospital, Centre South University, Changsha 410011, Hunan Province, China.

Received: 3 February 2016 Accepted: 15 March 2016

Published online: 22 March 2016

References

1. Mathers CD, Loncar D. Projections of global mortality and burden of disease from 2002 to 2030. PLoS Med. 2006;3:e442.

2. Mannino DM, Buist AS. Global burden of COPD: risk factors, prevalence, and future trends. Lancet. 2007;370:765-73.

3. $\operatorname{Sin} \mathrm{D}, \mathrm{Wy} L$, Man $\mathrm{S}$. The relationship between reduced lung function and cardiovascular mortality: a population-based study and a systemic review of the literature. Chest. 2005;127:1952-9.

4. Wolfrum C, Poy MN, Stoffel M. Apolipoprotein M is required for prebeta$\mathrm{HDL}$ formation and cholesterol efflux to $\mathrm{HDL}$ and protects against atherosclerosis. Nat Med. 2005;11:418-22.

5. Christoffersen C, Jauhiainen M, Moser M, Porse B, Ehnholm C, Boesl M, et al. Effect of apolipoprotein $\mathrm{M}$ on high density lipoprotein metabolism and atherosclerosis in low density lipoprotein receptor knock-out mice. J Biol Chem. 2008;283:1839-47.

6. Huang XS, Zhao SP, Hu M, Luo YP. Apolipoprotein M likely extends its anti-atherogenesis via anti-inflammation. Med Hypotheses. 2007;69:136-40. 
7. Elsoe S, Ahnstrom J, Christoffersen C, Hoofnagle AN, Plomgaard P, Heinecke JW, et al. Apolipoprotein $\mathrm{M}$ binds oxidized phospholipids and increases the antioxidant effect of HDL. Atherosclerosis. 2012;221:91-7.

8. Christoffersen C, Obinata H, Kumaraswamy SB, Galvani S, Ahnstrom J, Sevvana M, et al. Endothelium-protective sphingosine-1-phosphate provided by HDL-associated apolipoprotein M. Proc Natl Acad Sci U S A. 2011;108:9613-8.

9. Blaho VA, Galvani S, Engelbrecht E, Liu C, Swendeman SL, Kono M, et al. HDL-bound sphingosine-1-phosphate restrains lymphopoiesis and neuroinflammation. Nature. 2015;523:342-6.

10. Arkensteijn BW, Berbee JF, Rensen PC, Nielsen LB, Christoffersen C. The apolipoprotein $\mathrm{m}$-sphingosine-1-phosphate axis: biological relevance in lipoprotein metabolism, lipid disorders and atherosclerosis. Int J Mol Sci. 2013;14:4419-31.

11. Luo G, Zhang S, Nilsson-Ehle P, Xu N. Apolipoprotein M. Lipids Health Dis. 2004;3:21

12. Xu N, Nilsson-Ehle P, Hurtig M, Ahren B. Both leptin and leptin-receptor are essential for apolipoprotein M expression in vivo. Biochem Biophys Res Commun. 2004;321:916-21.

13. Xu N, Zhang XY, Dong X, Ekstrom U, Ye Q, Nilsson-Ehle P. Effects of platelet-activating factor, tumor necrosis factor, and interleukin-1alpha on the expression of apolipoprotein M in HepG2 cells. Biochem Biophys Res Commun. 2002;292:944-50.

14. Liang R, Zhang W, Song YM. Levels of leptin and IL-6 in lungs and blood are associated with the severity of chronic obstructive pulmonary disease in patients and rat models. Mol Med Rep. 2013;7:1470-6.

15. Shukla SD, Sohal SS, Mahmood MQ, Reid D, Muller HK, Walters EH. Airway epithelial platelet-activating factor receptor expression is markedly upregulated in chronic obstructive pulmonary disease. Int J Chron Obstruct Pulmon Dis. 2014;9:853-61.

16. Burkart KM, Manichaikul A, Wilk JB, Ahmed FS, Burke GL, Enright P, et al. APOM and high-density lipoprotein cholesterol are associated with lung function and per cent emphysema. Eur Respir J. 2014;43:1003-17.

17. Global Strategy for the Diagnosis, Management and Prevention of COPD, Global Initiative for Chronic Obstructive Lung Disease (GOLD). http://www.goldcopd.org/. Accessed 20 Oct 2015.

18. Clarenbach CF, Senn O, Sievi NA, Camen G, van Gestel AJ, Rossi VA, et al. Determinants of endothelial function in patients with COPD. Eur Respir J. 2013:42:1194-204

19. Clarenbach CF, Thurnheer R, Kohler M. Vascular dysfunction in chronic obstructive pulmonary disease: current evidence and perspectives. Expert Rev Respir Med. 2012;6:37-43.

20. Eickhoff P, Valipour A, Kiss D, Schreder M, Cekici L, Geyer K, et al. Determinants of systemic vascular function in patients with stable chronic obstructive pulmonary disease. Am J Respir Crit Care Med. 2008;178:1211-8.

21. Kumaraswamy SB, Linder A, Akesson P, Dahlback B. Decreased plasma concentrations of apolipoprotein $\mathrm{M}$ in sepsis and systemic inflammatory response syndromes. Crit Care. 2012;16:R60.

22. Feingold KR, Shigenaga JK, Chui LG, Moser A, Khovidhunkit W, Grunfeld C. Infection and inflammation decrease apolipoprotein M expression. Atherosclerosis. 2008;199:19-26.

23. Ciccone MM, Scicchitano P, Zito A, Cortese F, Boninfante B, Falcone VA, et al. Correlation between inflammatory markers of atherosclerosis and carotid intima-media thickness in Obstructive Sleep Apnea. Molecules. 2014;19:1651-62.

24. Ingersoll MA, Platt AM, Potteaux S, Randolph GJ. Monocyte trafficking in acute and chronic inflammation. Trends Immunol. 2011:32:470-7.

25. Garcia JG, Liu F, Verin AD, Birukova A, Dechert MA, Gerthoffer WT, et al. Sphingosine 1-phosphate promotes endothelial cell barrier integrity by Edg-dependent cytoskeletal rearrangement. J Clin Invest. 2001;108:689-701.

26. Gu JG, Zhu CL, Cheng DZ, Xie Y, Liu F, Zhou X. Enchanced levels of apolipoprotein $M$ during HBV infection feedback suppresses HBV replication. Lipids Health Dis. 2011;10:154.

27. Christoffersen C, Nielsen LB, Axler O, Andersson A, Johnsen AH, Dahlback B. Isolation and characterization of human apolipoprotein M-containing lipoproteins. J Lipid Res. 2006:47:1833-43.

28. Christoffersen C, Ahnstrom J, Axler O, Christensen El, Dahlback B, Nielsen LB. The signal peptide anchors apolipoprotein $M$ in plasma lipoproteins and prevents rapid clearance of apolipoprotein M from plasma. J Biol Chem. 2008;283:18765-72.
29. Khovidhunkit W, Memon RA, Feingold KR, Grunfeld C. Infection and inflammation-induced proatherogenic changes of lipoproteins. J Infect Dis. 2000;181 Suppl 3:S462-72.

30. Clifton PM, Mackinnon AM, Barter PJ. Effects of serum amyloid A protein (SAA) on composition, size, and density of high density lipoproteins in subjects with myocardial infarction. J Lipid Res. 1985;26:1389-98.

31. Memon RA, Staprans I, Noor M, Holleran WM, Uchida Y, Moser AH, et al. Infection and inflammation induce LDL oxidation in vivo. Arterioscler Thromb Vasc Biol. 2000;20:1536-42.

32. Feingold KR, Krauss RM, Pang M, Doerrler W, Jensen P, Grunfeld C. The hypertriglyceridemia of acquired immunodeficiency syndrome is associated with an increased prevalence of low density lipoprotein subclass pattern B. J Clin Endocrinol Metab. 1993;76:1423-7.

33. Koskinen J, Magnussen CG, Wurtz P, Soininen P, Kangas AJ, Viikari JS, et al. Apolipoprotein B, oxidized low-density lipoprotein, and LDL particle size in predicting the incidence of metabolic syndrome: the Cardiovascular Risk in Young Finns study. Eur J Prev Cardiol. 2012;19:1296-303.

34. Hurt-Camejo E, Camejo G, Rosengren B, Lopez F, Wiklund O, Bondjers G. Differential uptake of proteoglycan-selected subfractions of low density lipoprotein by human macrophages. J Lipid Res. 1990;31:1387-98.

35. Chait A, Brazg RL, Tribble DL, Krauss RM. Susceptibility of small, dense, low-density lipoproteins to oxidative modification in subjects with the atherogenic lipoprotein phenotype, pattern B. Am J Med. 1993;94:350-6.

36. Moore KJ, Tabas I. Macrophages in the pathogenesis of atherosclerosis. Cell. 2011;145:341-55.

37. Assmann G, Schulte $H$, von Eckardstein A, Huang Y. High-density lipoprotein cholesterol as a predictor of coronary heart disease risk. The PROCAM experience and pathophysiological implications for reverse cholesterol transport. Atherosclerosis. 1996;124(Suppl):S11-20.

38. Sharrett AR, Ballantyne CM, Coady SA, Heiss G, Sorlie PD, Catellier D, et al. Coronary heart disease prediction from lipoprotein cholesterol levels, triglycerides, lipoprotein(a), apolipoproteins A-I and B, and HDL density subfractions: The Atherosclerosis Risk in Communities (ARIC) Study. Circulation. 2001;104:1108-13.

39. Cardenas GA, Lavie CJ, Cardenas V, Milani RV, McCullough PA. The importance of recognizing and treating low levels of high-density lipoprotein cholesterol: a new era in atherosclerosis management. Rev Cardiovasc Med. 2008;9:239-58.

40. Emerging Risk Factors C, Erqou S, Kaptoge S, Perry PL, Di Angelantonio E, Thompson A, et al. Lipoprotein(a) concentration and the risk of coronary heart disease, stroke, and nonvascular mortality. JAMA. 2009;302:412-23.

41. Tsimikas S, Brilakis ES, Miller ER, McConnell JP, Lennon RJ, Kornman KS, et al. Oxidized phospholipids, Lp(a) lipoprotein, and coronary artery disease. N Engl J Med. 2005;353:46-57.

\section{Submit your next manuscript to BioMed Central and we will help you at every step:}

- We accept pre-submission inquiries

- Our selector tool helps you to find the most relevant journal

- We provide round the clock customer support

- Convenient online submission

- Thorough peer review

- Inclusion in PubMed and all major indexing services

- Maximum visibility for your research

Submit your manuscript at www.biomedcentral.com/submit 